\title{
Reduced-Complexity Coherent Versus Non-Coherent QAM-Aided Space-Time Shift Keying
}

\author{
Shinya Sugiura, Member, IEEE, Chao Xu, Student Member, IEEE, Soon Xin Ng, Senior Member, IEEE, \\ and Lajos Hanzo, Fellow, IEEE
}

\begin{abstract}
A novel reduced-complexity near-optimal detection algorithm is proposed for enhancing the recent Coherentlydetected Space-Time Shift Keying (CSTSK) scheme employing arbitrary constellations, such as $\mathcal{L}$-point Phase-Shift Keying (PSK) and Quadrature Amplitude Modulation (QAM). The proposed detector relies on a modified Matched Filter (MF) concept. More specifically, we exploit both the constellation diagram of the modulation scheme employed as well as the Inter-ElementInterference (IEI)-free STSK architecture. Furthermore, we generalize the Pulse Amplitude Modulation (PAM)- or PSK-aided Differentially-encoded STSK (DSTSK) concept and conceive its more bandwidth-efficient QAM-aided counterpart. Then, the proposed reduced-complexity CSTSK detector is applied to the QAM-aided DSTSK scheme, which enables us to carry out low-complexity non-coherent detection, while dispensing with channel estimation. It is revealed that the proposed detector is capable of approaching the optimal Maximum Likelihood (ML) detector's performance, while avoiding the exhaustive ML search. Interestingly, our simulation results also demonstrate that the reduced-complexity detector advocated may achieve the same performance as that of the optimal ML detector for the specific STSK scheme's parameters. Another novelty of this paper is that the star-QAM STSK scheme tends to outperform its square-QAM counterpart, especially for high number of dispersion matrices. Furthermore, we provided both the theoretical analysis and the simulations, in order to support this unexpected fact.
\end{abstract}

Index Terms-Differential encoding, diversity and multiplexing tradeoff, space-time shift keying, spatial modulation, matched filter, multiple antenna array, non-coherent detection.

\section{INTRODUCTION}

$\mathbf{T}$ HE recent Multiple-Input Multiple-Output (MIMO) concept of Spatial Modulation (SM) [1]-[4] or SpaceShift Keying (SSK) [5]-[7] is capable of increasing the achievable transmission rate with the aid of multiple-antenna aided systems, which is ensured without resorting to spatial multiplexing [8], [9]. To be more specific, since the SM

Paper approved by M. Juntti, the Editor for MIMO and Multiple-Access of the IEEE Communications Society. Manuscript received March 9, 2011; revised June 15, 2011.

The work of S. Sugiura was partially supported by KAKENHI (23760353). The financial support of the RC-UK under the auspices of the India-UK Advanced Technology Centre, as well as of the China-UK $4^{\text {th }}$ generation wireless systems project and that of the European Union under the auspices of the Optimix project, is gratefully acknowledged.

S. Sugiura is with Toyota Central R\&D Laboratories, Inc., Aichi, 480-1192, Japan (e-mail: sugiura@mosk.tytlabs.co.jp).

C. Xu, S. X. Ng, and L. Hanzo are with the School of Electronics and Computer Science, University of Southampton, Southampton, SO17 1BJ, UK (e-mail: $\{$ cx1g08, sxn, lh $\} @$ ecs.soton.ac.uk).

Digital Object Identifier 10.1109/TCOMM.2011.100411.110154 transmitter activates one out of $M$ antenna elements for conveying additional information bits during each symbol interval, no Inter-Element-Interference (IEI) is imposed on the receiver, hence potentially enabling low-complexity singlestream detection. Due to the different system architecture of classic spatial multiplexing and of the SM/SSK schemes, new detection algorithms specific to the SM/SSK schemes have been developed, which may be classified into two fundamental categories, namely the low-complexity MatchedFilter (MF) based detector [1] and the single-antenna-based optimal Maximum-Likelihood (ML) detector [2]. In practice, the majority of the previous SM/SSK receivers have adopted the single-stream ML detector [2]-[7], where the optimal BER performance is achieved at the cost of an increased decoding complexity.

On the other hand, the MF-based detector [1] exhibits a significantly reduced complexity, since the antenna index $m$ and the modulated constellation point $l$ are separately estimated. However, as mentioned in [2], [10], this sub-optimal detector only works under the idealized assumption of encountering noiseless channels at the antenna-index estimation stage. Recently, the novel concept of Space-Time Shift Keying (STSK) has been proposed in [11]-[13], where the encoding principle is characterized by the fact that one out of $Q$ space-time dispersion matrices is selected, while the abovementioned SM and SSK schemes simply activates one out of $M$ antenna elements. Since the STSK scheme is capable of exploiting both the space- and time-dimensions, it allows us to strike a flexible balance between the maximum attainable diversity order and the throughput. ${ }^{1}$ Previous studies of the STSK scheme [11]-[13] also considered the optimal singlestream-based ML detector, similarly to the SM/SSK schemes [2]. One exception is constituted by the solution in [14], where a reduced-complexity detection algorithm was developed in the context of Differentially-encoded STSK (DSTSK) systems [11], which was assisted by Multiple-Symbol Differential Sphere Decoding (MSDSD). ${ }^{2}$ The introduction of differen-

\footnotetext{
${ }^{1}$ In order to expound a little further, both the SM and SSK schemes are subsumed by the STSK arrangement, as demonstrated in [11], [13].

${ }^{2}$ In [15] a Sphere Detector (SD) was conceived for the SM scheme, in order to closely approximate the optimal ML search. Furthermore, in [14] the matched filter based non-coherent detector was combined with a SD in the context of a differentially-encoded STSK scheme, for the sake of further reducing the conventional SD's complexity without imposing any substantial performance degradation. Although the same idea may be readily applicable to the detector proposed in this paper, the related investigations will be left for our future studies.
} 
tial encoding was an important mile-stone, since the corresponding low-complexity non-coherent receivers are capable of outperforming their high-complexity coherently detected counterparts in the presence of realistic pilot-aided channel estimations, as detailed in [16], [17]. However, the applicability of this detector is limited to specific low-order constellations, such as On-Off Keying (OOK), Binary Phase-Shift Keying (BPSK), Quadrature PSK (QPSK) and 8-PSK. Regretfully, it is not suitable for bandwidth-efficient Quadrature Amplitude Modulation (QAM) [18].

Against this background, the novel contributions of this paper are as follows:

- Two efficient near-optimal detectors are proposed for the Coherently-detected STSK (CSTSK) scheme. More specifically, both detectors rely on a modified MF concept, while taking into account the particular constellation diagram of the modulation scheme employed. To be more specific, while the first detector invokes exhaustive signalspace search at the MF's output, the second detector further reduces the first detector's complexity at the cost of a modest performance penalty. The proposed detectors are applicable to CSTSK receiver employing arbitrary modulation schemes, including high-order QAM. Furthermore, since the CSTSK scheme subsumes the family of SM/SSK schemes as its special case, the proposed low-complexity detector is directly applicable to the class of SM/SSK schemes. Also, the computational complexity imposed by the proposed detector is quantified and compared to that of the conventional detectors in [1] and [2].

- Since our previous DSTSK schemes were limited to Pulse Amplitude Modulation (PAM) [11] and PSK [14], we extend them to their higher-throughput QAM-aided counterpart. Then, the new reduced-complexity detectors proposed for CSTSK in this paper are further developed to its non-coherently detected QAM-aided DSTSK counterpart. The advantage of the non-coherent DSTSK detector over its coherent counterpart is quantified in scenarios contaminated by channel-estimation errors.

- An additional result of this paper is that the star-QAM assisted STSK scheme tends to outperform its squareQAM aided counterpart. This holds true both for the optimal ML and for the proposed near-optimal detectors. We carried out both the related analysis as well as computer simulations, in order to support this somewhat unexpected fact.

The remainder of this paper is organized as follows. Section II outlines the system model of the CSTSK scheme. In Section III we commence by reviewing the conventional detectors and propose the novel near-optimal reduced-complexity detection schemes, while Section IV applies the proposed detectors to the non-coherent STSK scenario and then Section V provides our simulation results. Finally, Section VI concludes this paper.

\section{System Model of Coherent STSK}

In this section, we briefly review the encoding principle and the received signal model of the CSTSK scheme [11]. At the CSTSK transmitter, information bits are encoded with the aid of two different operations, namely the dispersionmatrix activation and the classic $\mathcal{L}$-PSK/QAM modulation. More specifically, the $Q$ space-time dispersion matrices $\mathbf{A}_{q} \in \mathcal{C}^{M \times T}(q=1, \cdots, Q)$ satisfying the power-constraint relationship of $\operatorname{tr}\left(\mathbf{A}_{q}^{H} \mathbf{A}_{q}\right)=T$ are assigned to the transmitter in advance of transmissions, where $M$ and $T$ denote the number of transmit antennas and the number of symbols per space-time block duration, respectively. Firstly, $B=$ $\log _{2}(Q \cdot \mathcal{L})$ information bits per space-time block are input to the transmitter, and then the input bits are Serial-to-Parallel (S/P) converted to $B_{1}=\log _{2} Q$ and $B_{2}=\log _{2} \mathcal{L}$ bits. Next, one out of $Q$ dispersion matrices is selected as $\mathbf{A}_{q}$ according to the $B_{1}=\log _{2} Q$ input bits, while the $B_{2}=\log _{2} \mathcal{L}$ input bits are modulated to a PSK/QAM symbol $s_{l}$. Finally, a spacetime codeword $\mathbf{S}_{q, l}=s_{l} \mathbf{A}_{q} \in \mathcal{C}^{M \times T}$ is transmitted from the $M$ transmit antenna elements over $T$ symbol durations.

The corresponding block-based signals $\mathbf{Y} \in \mathcal{C}^{N \times T}$ received at the CSTSK receiver may be expressed as

$$
\mathbf{Y}=\mathbf{H S}+\mathbf{N},
$$

where $\mathbf{H} \in \mathcal{C}^{N \times M}$ represent the channel components, each obeying the complex-valued Gaussian distribution having a zero mean and a unity variance, i.e. $\mathcal{C N}(0,1)$, while each noise element of $\mathbf{N} \in \mathcal{C}^{N \times T}$ is the complex-valued Gaussian variable obeying $\mathcal{C N}\left(0, N_{0}\right)$. Furthermore, $N$ denotes the number of receive antennas and $N_{0}$ represents the noise variance.

By implementing the vectorial stacking operation $\operatorname{vec}(\cdot)$ at both sides of Eq. (1) as shown in [11], we arrive at the equivalent system model as

$$
\overline{\mathbf{Y}}=\overline{\mathbf{H}} \mathbf{K}_{q, l}+\overline{\mathbf{N}},
$$

where we have

$$
\begin{aligned}
\overline{\mathbf{Y}} & =\operatorname{vec}(\mathbf{Y}) \in \mathcal{C}^{N T \times 1} \\
\overline{\mathbf{H}} & =\left[\overline{\mathbf{h}}_{1}, \cdots, \overline{\mathbf{h}}_{Q}\right] \\
& =\left(\mathbf{I}_{T} \otimes \mathbf{H}\right) \chi \in \mathcal{C}^{N T \times Q} \\
\chi & =\left[\operatorname{vec}\left(\mathbf{A}_{1}\right), \cdots, \operatorname{vec}\left(\mathbf{A}_{Q}\right)\right] \in \mathcal{C}^{M T \times Q} \\
\overline{\mathbf{N}} & =\operatorname{vec}(\mathbf{N}) \in \mathcal{C}^{N T \times 1}
\end{aligned}
$$

and

$$
\begin{gathered}
\mathbf{K}_{q, l}=\left[0, \cdots, 0, s_{l}, 0, \cdots, 0\right]^{\mathrm{T}} \in \mathcal{C}^{Q \times 1} . \\
\uparrow \\
q \text { th element }
\end{gathered}
$$

Here, $\otimes$ represents the Kronecker product operation. In the rest of this paper, we employ the parametric notation of ' $\operatorname{STSK}(M, N, T, Q)$ ' for the sake of space economy.

\section{LOW-COMLEXITY MF-BASED CSTSKS}

This section firstly introduces the two conventional detectors in the context of the CSTSK arrangement, namely the conventional MF-based detector [1] and the single-antennnabased ML detector [2]. Then we continue by outlining the two near-optimal receiver architectures advocated, which exploits the properties of the $\mathcal{L}$-PSK/QAM constellations employed. Furthermore, we compare the computational complexity imposed by these four detectors. Note that the aim of the CSTSK detectors is to identify the transmitted index set $(q, l)$ in a reliable and low-complexity manner. 


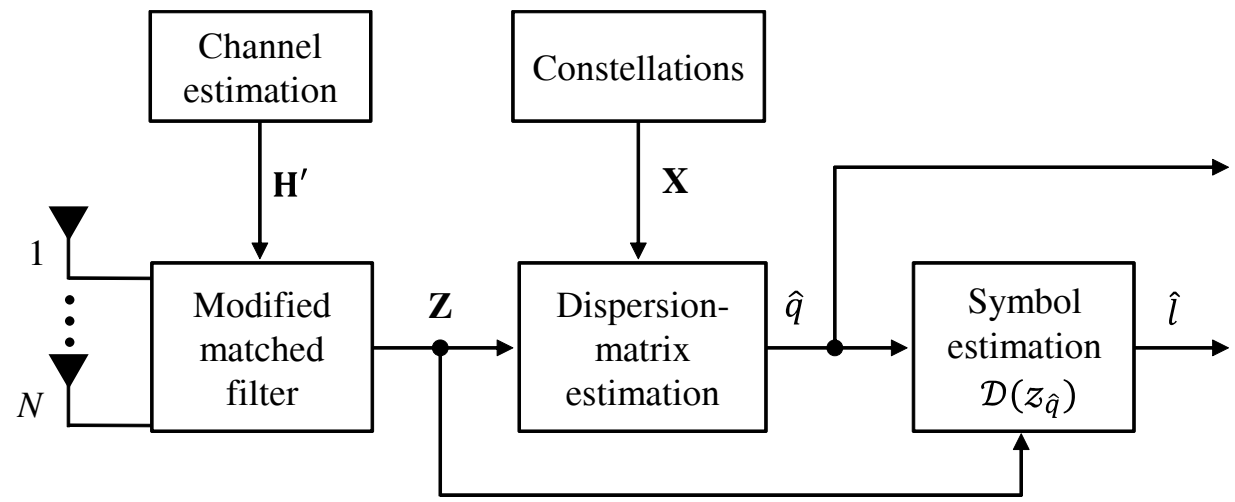

Fig. 1. The structure of the proposed Detectors I and II.

\section{A. The Conventional MF-Based Detector [1]}

In the conventional detector of [1], the Hermitian transpose of the equivalent channel matrix $\overline{\mathbf{H}}$ is multiplied by the equivalent received signal $\overline{\mathbf{Y}}$ in order to formulate the decision metric of $\mathbf{G}=\left[g_{1}, \cdots, g_{Q}\right]^{\mathrm{T}} \in \mathcal{C}^{Q \times 1}$, as follows:

$$
\mathbf{G}=\overline{\mathbf{H}}^{H} \overline{\mathbf{Y}} \text {. }
$$

Then, the index $q$ of the activated dispersion matrix and the transmitted symbol index $l$ are estimated separately, as follows:

$$
\begin{aligned}
\hat{q} & =\arg \max _{q}\left|g_{q}\right|, \\
\hat{l} & =\mathcal{D}\left(g_{\hat{q}}\right)=\arg \min _{l}\left|g_{\hat{q}}-\left\|\mathbf{h}_{\hat{q}}\right\|^{2} s_{l}\right|,
\end{aligned}
$$

where $\mathcal{D}$ denotes the demodulation function.

Note that since the transmitted-symbol estimation process of Eq. (10) includes the result of the dispersion-matrix estimation formulated in Eq. (9), the potential mis-detection of $\hat{q}$ may induce error propagation. Importantly, while the search space of this detector determined by Eqs. (9) and (10), is as low as the order of $(Q+\mathcal{L})$, this detection scheme tends to exhibit an error floor in fading environments [2], [10]. This is mainly due to the fact that low-complexity MF-operation of Eq. (8) ignores the effect of the channel's fading envelope, as well as because the decision metric of Eq. (9) only considers the absolute value of the matched-filtered symbol, rather than individually considering each constellation point. In Section III-C below, our detector will be further developed for the sake of combating these limitations, hence ultimately achieving a higher BER performance than that of the detector of this section.

\section{B. The Single-Stream-Based ML Detector [2], [11]}

The optimal ML performance may be attained by implementing exhaustive search over the legitimate CSTSK codewords $\mathbf{K}_{q, l}(1 \leq q \leq Q, 1 \leq l \leq \mathcal{L})$ at the cost of an increased complexity. To be more specific, by maximizing the probability of $P\left(\overline{\mathbf{Y}} \mid \overline{\mathbf{H}}, \mathbf{K}_{q, l}\right)$, the ML search may be expressed as

$$
\begin{aligned}
(\hat{q}, \hat{l}) & =\arg \min _{(q, l)}\left\|\overline{\mathbf{Y}}-\overline{\mathbf{H}} \mathbf{K}_{q, l}\right\|^{2} \\
& =\arg \min _{(q, l)}\left\|\overline{\mathbf{Y}}-s_{l} \overline{\mathbf{h}}_{q}\right\|^{2},
\end{aligned}
$$

where $\overline{\mathbf{h}}_{q}$ is the $q$ th column of $\overline{\mathbf{H}}$. As shown in Eq. (12), the search space size of the ML detector is the order of $(Q \cdot \mathcal{L})$, which is higher than $(Q+\mathcal{L})$ in the detector [1] outlined in Section III-A.

\section{The Proposed MF-Based Detector I}

In this section, we present the new reduced-complexity CSTSK detector of Fig. 1. According to Eq. (4), the estimated channels $\mathbf{H}$ are firstly transformed to the equivalent channels $\overline{\mathbf{H}}$. Then, we normalized each column of $\overline{\mathbf{H}}$ in order to generate the modified equivalent channels $\mathbf{H}^{\prime}$ as ${ }^{3}$

$$
\mathbf{H}^{\prime}=\left[\frac{\overline{\mathbf{h}}_{1}}{\left\|\overline{\mathbf{h}}_{1}\right\|}, \cdots, \frac{\overline{\mathbf{h}}_{Q}}{\left\|\overline{\mathbf{h}}_{Q}\right\|}\right] .
$$

Then, we have the MF output of

$$
\mathbf{Z}=\left[z_{1}, \cdots, z_{Q}\right]^{\mathrm{T}}=\mathbf{H}^{\prime H} \overline{\mathbf{Y}} .
$$

Next, we consider the vector-by-vector exhaustive $(q, l)$ search for the MF-output signals of $\mathbf{Z}$ as follows:

$$
\begin{aligned}
(\hat{q}, \hat{l})= & \arg \min _{q . l}\|\mathbf{Z}-\| \overline{\mathbf{h}}_{q}\left\|\mathbf{K}_{q, l}\right\|^{2} \\
= & \arg \min _{q . l}\left(\left|z_{q}-\left\|\overline{\mathbf{h}}_{q}\right\| s_{l}\right|^{2}+\sum_{q^{\prime} \neq q}\left|z_{q^{\prime}}\right|^{2}\right) \\
= & \arg \min _{q . l}\left(\left|z_{q}-\left\|\overline{\mathbf{h}}_{q}\right\| s_{l}\right|^{2}+\|\mathbf{Z}\|^{2}-\left|z_{q}\right|^{2}\right) \\
= & \arg \max _{q . l}\left(\left|z_{q}\right|^{2}-\left|z_{q}-\left\|\overline{\mathbf{h}}_{q}\right\| s_{l}\right|^{2}\right) \\
= & \arg \max _{q . l}\left[2\left\|\overline{\mathbf{h}}_{q}\right\|\left\{\Re\left(z_{q}\right) \Re\left(s_{l}\right)+\Im\left(z_{q}\right) \Im\left(s_{l}\right)\right\}\right. \\
& \left.-\left\|\overline{\mathbf{h}}_{q}\right\|^{2}\left|s_{l}\right|^{2}\right]
\end{aligned}
$$

while $\Re(\cdot)$ and $\Im(\cdot)$ represent the real and imaginary part, respectively. In order to reduce the complexity of the detection relying on Eq. (19), we introduce the separate detection of $q$ and $l$. More specifically, assuming that the modulation scheme $s_{l}$ has symmetric properties around both the $I$ - and $Q$-axis, the detection of the activated dispersion-matrix index $q$ can be expressed from Eq. (19) as Eqs. (20) and (21) at the top

\footnotetext{
${ }^{3}$ The computations of the modified equivalent channels $\mathbf{H}^{\prime}$ are carried out as a part of the detection process in our detector, which has to be updated at intervals corresponding to the channel's coherence time $\tau$. This naturally imposes an additional decoding complexity and hence will be taken into account in the complexity evaluation of Section III-E.
} 


$$
\begin{aligned}
\hat{q}= & \arg \max _{q, \forall l^{\prime}}\left[2\left\|\overline{\mathbf{h}}_{q}\right\|\left\{ \pm\left|\Re\left(z_{q}\right)\right|\left|\Re\left(s_{l^{\prime}}^{\prime}\right)\right| \pm\left|\Im\left(z_{q}\right)\right|\left|\Im\left(s_{l^{\prime}}^{\prime}\right)\right|\right\}\right. \\
& \left.-\left\|\overline{\mathbf{h}}_{q}\right\|^{2}\left|s_{l^{\prime}}^{\prime}\right|^{2}\right] \\
= & \arg \max _{q, \forall l^{\prime}}\left[2\left\|\overline{\mathbf{h}}_{q}\right\|\left\{\left|\Re\left(z_{q}\right)\right|\left|\Re\left(s_{l^{\prime}}^{\prime}\right)\right|+\left|\Im\left(z_{q}\right)\right|\left|\Im\left(s_{l^{\prime}}^{\prime}\right)\right|\right\}\right. \\
& \left.-\left\|\overline{\mathbf{h}}_{q}\right\|^{2}\left|s_{l^{\prime}}^{\prime}\right|^{2}\right]
\end{aligned}
$$

of the next page, where $s_{l^{\prime}}^{\prime}\left(l^{\prime}=1, \cdots, \mathcal{L}^{\prime}\right)$ represents the constellation points, which corresponds to the points $s_{l}$ having the relationship of $\Re\left(s_{l}\right), \Im\left(s_{l}\right) \geq 0$, as shown in Fig. 2.4 Then, by using the dispersion-matrix index $\hat{q}$ estimated in Eq. (21), the symbol index $l$ is detected according to

$$
\hat{l}=\mathcal{D}\left(z_{\hat{q}}\right)=\arg \min _{l}\left|z_{\hat{q}}-\left\|\overline{\mathbf{h}}_{\hat{q}}\right\| s_{l}\right| .
$$

Note that the legitimate search space of Eqs. (21) and (22) is $Q \mathcal{L}^{\prime}(\simeq Q \mathcal{L} / 4)$ and $\mathcal{L}$, respectively, which is typically smaller than the search space $Q \mathcal{L}$ of Eq. (19). Here, we define the detector proposed in this section as 'Detector I', which is characterized by Eqs. (14), (21) and (22).

\section{The Proposed MF-Based Detector II}

Furthermore, in this section we propose another detector, which is capable of further reducing the complexity of Detector I of Section III-C. To be specific, the $q$-detection part of Eq. (21) in Detector I is further approximated, while allowing a modest performance penalty. Firstly, Eq. (21) is rewritten as: Eq. (23), which is shown at the top of the following page.

Then, by only considering the last term of Eq. (23), we arrive at its approximation of

$$
\hat{q}=\arg \max _{q, \forall l^{\prime}}\left[\left|\Re\left(z_{q}\right)\right| \frac{\left|\Re\left(s_{l^{\prime}}^{\prime}\right)\right|}{\left|s_{l^{\prime}}^{\prime}\right|}+\left|\Im\left(z_{q}\right)\right| \frac{\left|\Im\left(s_{l^{\prime}}^{\prime}\right)\right|}{\left|s_{l^{\prime}}^{\prime}\right|}\right] .
$$

Here, let us define the legitimate pairs of $\left(\left|\Re\left(s_{l^{\prime}}^{\prime}\right)\right| /\left|s_{l^{\prime}}^{\prime}\right|,\left|\Im\left(s_{l^{\prime}}^{\prime}\right)\right| /\left|s_{l^{\prime}}^{\prime}\right|\right)$ as $\mathrm{V}$ number of unit-norm vectors $\mathbf{x}_{v}=\left[x_{v, I}, x_{v, Q}\right](v=1, \cdots, V)$. More explicitly, as exemplified in Fig. 2 for both 16 square-QAM and 16 star-QAM, we have $V=3$ vectors. $^{5}$ Finally, we have the following decision metric of

$$
\begin{aligned}
\hat{q} & =\arg \max _{q, \forall v}\left(g_{q, v}\right) \\
& =\arg \max _{q, \forall v}\left[\left|\Re\left(z_{q}\right)\right| x_{v, I}+\left|\Im\left(z_{q}\right)\right| x_{v, Q}\right] .
\end{aligned}
$$

Finally, the approximated version of Detector I is constituted by Eqs. (14), (26) and (22), which is referred to as 'Detector

\footnotetext{
${ }^{4}$ For example, $s_{l^{\prime}}^{\prime}\left(l^{\prime}=1, \cdots, L\right)^{\prime}$ is given by $s_{l^{\prime}}^{\prime} \in\{1\}$ for BPSK, while we have $s_{l^{\prime}}^{\prime} \in\{1 / \sqrt{2}+j / \sqrt{2}\}$ for QPSK, $s_{l^{\prime}}^{\prime} \in\{1,1 / \sqrt{2}+j / \sqrt{2}, j\}$ for 8 -PSK and $s_{l^{\prime}}^{\prime} \in\{1 / \sqrt{10}+j / \sqrt{10}, 1 / \sqrt{10}+j 3 / \sqrt{10}, 3 / \sqrt{10}+$ $j / \sqrt{10}, 3 / \sqrt{10}+j 3 / \sqrt{10}\}$ for 16 square-QAM. Similarly, $s_{l^{\prime}}^{\prime}\left(l^{\prime}=\right.$ $1, \cdots, L)^{\prime}$ can be defined for any of the modulation schemes, which have symmetric properties around the $I$ - and $Q$-axis.

${ }^{5}$ To elaborate a little further, this process of generating $\mathbf{x}_{v}(v=1, \cdots, V)$ from the constellation points is applicable to modulation schemes, which have symmetric properties around both the $s_{I}$ and $s_{Q}$ axes. This indicates that the proposed detection may be used for most of the conventional PSK/QAM schemes or other constellation schemes, which do not have such symmetric properties, all the constellation points have to be considered for the calculation of $\mathbf{x}_{v}(v=1, \cdots, V)$. Naturally, this is only possible at the cost of an increased value of $\mathrm{V}$, hence an increased decoding complexity, as formulated in Eq. (35) of Section III-E.
}

II' in this paper. For more details, we listed in Table I a set of examples for characterizing the mapping of classic PSK/QAM symbols to the corresponding sets of $\mathbf{x}_{v}(v=1, \cdots, V){ }^{6}$

To be more specific, given a transmitted index set of $(q, l)$ and the corresponding value of $v$, the correct element $g_{q, v}^{\prime}$ of the decision metric $\mathbf{G}^{\prime}$ is represented by

$$
\begin{aligned}
g_{q, v}^{\prime} & =x_{v, I}\left|\left\|\overline{\mathbf{h}}_{q}\right\| \Re\left(s_{l}\right)+\Re\left(\frac{\overline{\mathbf{h}}_{q}^{H}}{\left\|\overline{\mathbf{h}}_{q}\right\|} \overline{\mathbf{N}}\right)\right| \\
& +x_{v, Q}\left|\left\|\overline{\mathbf{h}}_{q}\right\| \Im\left(s_{l}\right)+\Im\left(\frac{\overline{\mathbf{h}}_{q}^{H}}{\left\|\overline{\mathbf{h}}_{q}\right\|} \overline{\mathbf{N}}\right)\right|,
\end{aligned}
$$

while the incorrect element $g_{\tilde{q}, v^{\prime}}^{\prime}\left(\tilde{q} \neq q, 1 \leq v^{\prime} \leq V\right)$ may be expressed as

$$
\begin{aligned}
& g_{\tilde{q}, v^{\prime}}^{\prime}=x_{v^{\prime}, I}\left|\Re\left(\frac{\overline{\mathbf{h}}_{\tilde{q}}^{H}}{\left\|\overline{\mathbf{h}}_{\tilde{q}}\right\|} \overline{\mathbf{h}}_{q} s_{l}\right)+\Re\left(\frac{\overline{\mathbf{h}}_{\tilde{q}}^{H}}{\left\|\overline{\mathbf{h}}_{\tilde{q}}\right\|} \overline{\mathbf{N}}\right)\right| \\
& +\quad x_{v^{\prime}, Q}\left|\Im\left(\frac{\overline{\mathbf{h}}_{\tilde{q}}^{H}}{\left\|\overline{\mathbf{h}}_{\tilde{q}}\right\|} \overline{\mathbf{h}}_{q} s_{l}\right)+\Im\left(\frac{\overline{\mathbf{h}}_{\tilde{q}}^{H}}{\left\|\overline{\mathbf{h}}_{\tilde{q}}\right\|} \overline{\mathbf{N}}\right)\right| .
\end{aligned}
$$

Provided that we have

$$
g_{q, v}^{\prime}>g_{\tilde{q}, v^{\prime}}^{\prime} \quad\left(q \neq \tilde{q}, 1 \leq v^{\prime} \leq V\right),
$$

the correct index $q$ is found during each block interval. Here, let us consider the ultimate scenario of $N_{0} \rightarrow 0$. Then Eq. (29) becomes

$$
\begin{array}{r}
\left\|\overline{\mathbf{h}}_{q}\right\| \underbrace{\left\{x_{v, I}\left|\Re\left(s_{l}\right)\right|+x_{v, Q}\left|\Im\left(s_{l}\right)\right|\right\}}_{\phi}> \\
x_{v^{\prime}, I}\left|\Re\left(\frac{\overline{\mathbf{h}}_{\tilde{q}}^{H}}{\left\|\overline{\mathbf{h}}_{\tilde{q}}\right\|} \overline{\mathbf{h}}_{q} s_{l}\right)\right|+x_{v^{\prime}, Q}\left|\Im\left(\frac{\overline{\mathbf{h}}_{\tilde{q}}^{H}}{\left\|\overline{\overline{\mathbf{h}}}_{\tilde{q}}\right\|} \overline{\mathbf{h}}_{q} s_{l}\right)\right| .
\end{array}
$$

We note that considering the relationships of $\left|x_{v^{\prime}, I}\right|^{2}+$ $\left|x_{v^{\prime}, Q}\right|^{2}=1$ as well as of $\left|\Re\left(s_{l}\right)\right|,\left|\Im\left(s_{l}\right)\right| \geq 0, \phi=$ $x_{v, I}\left|\Re\left(s_{l}\right)\right|+x_{v, Q}\left|\Im\left(s_{l}\right)\right|$ of Eq. (30) may be upper-bounded by

$$
\begin{aligned}
\phi= & x_{v, I}\left|\Re\left(s_{l}\right)\right|+x_{v, Q}\left|\Im\left(s_{l}\right)\right| \\
= & \sqrt{\left|\Re\left(s_{l}\right)\right|^{2}+\left|\Im\left(s_{l}\right)\right|^{2}} \cdot\left\{x_{v, I} \frac{\left|\Re\left(s_{l}\right)\right|}{\sqrt{\left|\Re\left(s_{l}\right)\right|^{2}+\left|\Im\left(s_{l}\right)\right|^{2}}}\right. \\
& \left.+x_{v, Q} \frac{\left|\Im\left(s_{l}\right)\right|}{\sqrt{\left|\Re\left(s_{l}\right)\right|^{2}+\left|\Im\left(s_{l}\right)\right|^{2}}}\right\} \\
\leq & \sqrt{\left|\Re\left(s_{l}\right)\right|^{2}+\left|\Im\left(s_{l}\right)\right|^{2}}=\left|s_{l}\right| .
\end{aligned}
$$

${ }^{6}$ Furthermore, for $(\mathcal{L} \geq 4)$-point PSK constellations, we may be able to formulate a set of parameters $\left(\mathbf{x}_{v}, V\right)$ as $\mathbf{x}_{v}=\left[\cos \frac{\pi(v-1)}{2(V-1)} \sin \frac{\pi(v-1)}{2(V-1)}\right](v=$ $1, \cdots, V)$ as well as $V=\mathcal{L} / 4+1$. 


$$
\begin{array}{r}
\hat{q}=\arg \max _{q, \forall l^{\prime}}\left[2\left\|\overline{\mathbf{h}}_{q}\right\|\left\{\left|\Re\left(z_{q}\right)\right| \frac{\left|\Re\left(s_{l^{\prime}}^{\prime}\right)\right|}{\left|s_{l^{\prime}}^{\prime}\right|}+\left|\Im\left(z_{q}\right)\right| \frac{\left|\Im\left(s_{l^{\prime}}^{\prime}\right)\right|}{\left|s_{l^{\prime}}^{\prime}\right|}\right\}\left|s_{l^{\prime}}^{\prime}\right|-|| \overline{\mathbf{h}}_{q} \|^{2}\left|s_{l^{\prime}}^{\prime}\right|^{2}\right], \\
=\arg \max _{q, \forall l^{\prime}}\left[-\left(\left\|\overline{\mathbf{h}}_{q}\right\||| s_{l^{\prime}}^{\prime} \mid-\left\{\left|\Re\left(z_{q}\right)\right| \frac{\left|\Re\left(s_{l^{\prime}}^{\prime}\right)\right|}{\left|s_{l^{\prime}}^{\prime}\right|}+\left|\Im\left(z_{q}\right)\right| \frac{\left|\Im\left(s_{l^{\prime}}^{\prime}\right)\right|}{\left|s_{l^{\prime}}^{\prime}\right|}\right\}\right)^{2}+\left\{\left|\Re\left(z_{q}\right)\right| \frac{\left|\Re\left(s_{l^{\prime}}^{\prime}\right)\right|}{\left|s_{l^{\prime}}^{\prime}\right|}+\left|\Im\left(z_{q}\right)\right| \frac{\left|\Im\left(s_{l^{\prime}}^{\prime}\right)\right|}{\left|s_{l^{\prime}}^{\prime}\right|}\right\}^{2}\right]
\end{array}
$$

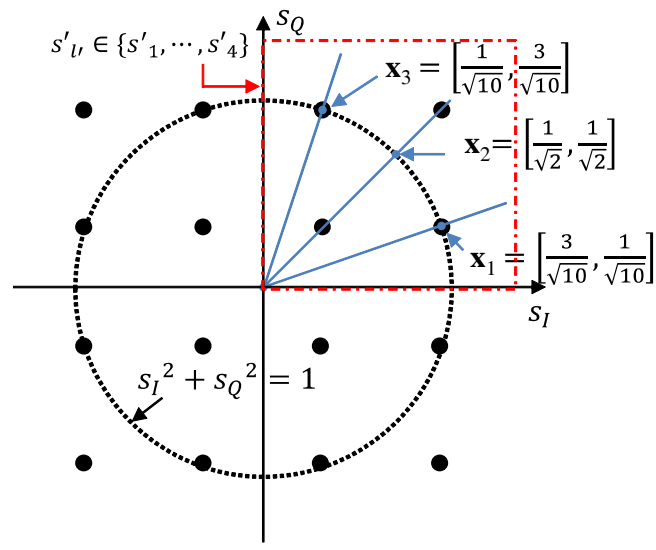

16 square QAM

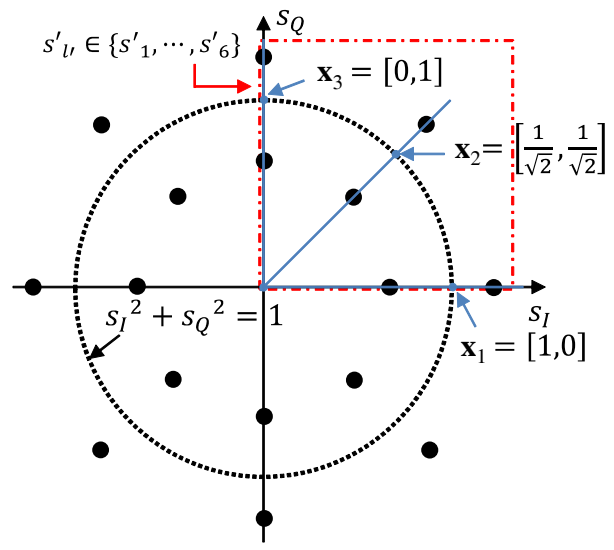

16 star QAM

Fig. 2. Sets of $s_{l^{\prime}}^{\prime} \in\left\{s_{1}^{\prime}, \cdots, s_{\mathcal{L}^{\prime}}^{\prime}\right\}$ and $\mathbf{X}=\left[\mathbf{x}_{1}^{\mathrm{T}}, \cdots, \mathbf{x}_{V}^{\mathrm{T}}\right]$ for 16 square-QAM and star-QAM constellations.

TABLE I

MAPPing EXAmples From Modulation SChemes to Points $\mathbf{x}_{v}(v=1, \cdots, V)$

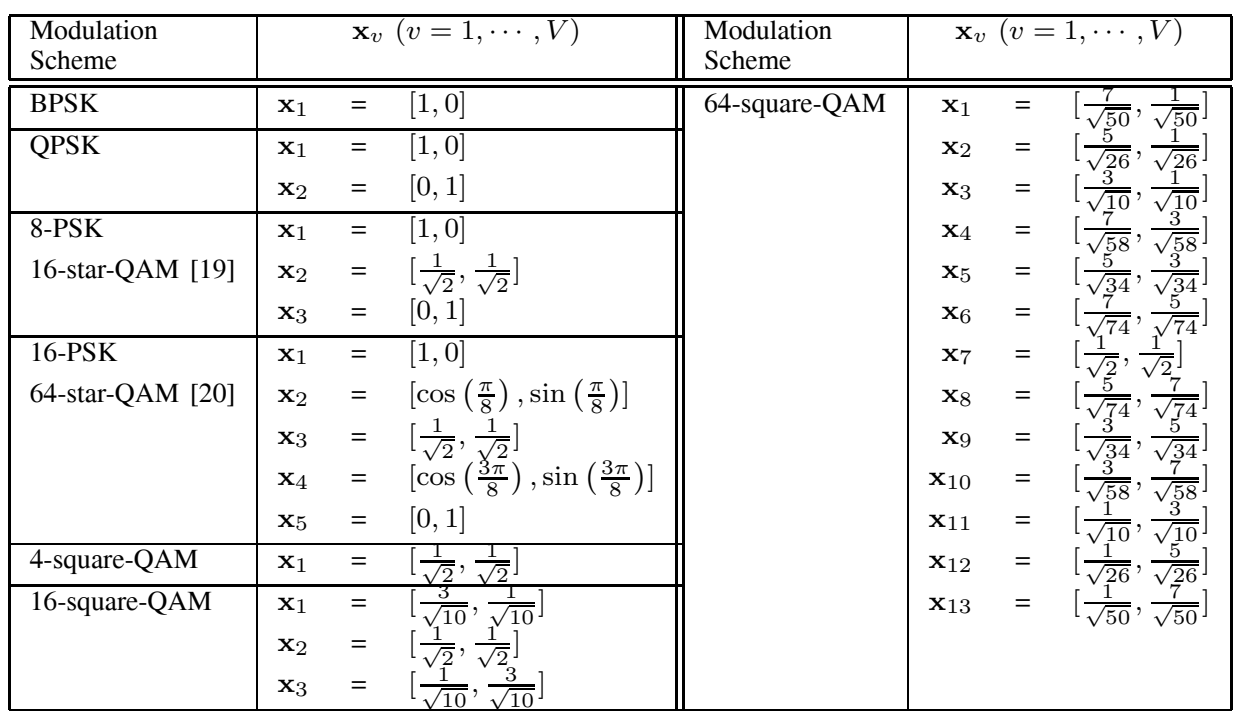

In Eq. (31) we have equality if and only if

$$
\frac{x_{v, I}}{x_{v, Q}}=\frac{\left|\Re\left(s_{l}\right)\right|}{\left|\Im\left(s_{l}\right)\right|} .
$$

Therefore, the projection operation from the constellation points $s_{l}(1 \leq l \leq \mathcal{L})$ to the corresponding points $\mathbf{x}_{v}(1 \leq$ $v \leq V)$ on the unit circle, as shown in Fig. 2, is aimed for having $\mathbf{x}_{v}$, which satisfies Eq. (32), hence maximizes the left hand side of Eq. (30). As a result, Eq. (30) is always satisfied and the conventional detector's error floor can be eliminated.

\section{E. Complexity Analysis}

In this section, we compare the computational complexity imposed by the four detectors presented in Section III, i.e. the original MF-based detector [1], the optimal ML detector [2], the proposed Detector I of Section III-C and the proposed Detector II of Section III-D. Here, we quantified the complexity as the number of real-valued multiplications, where a single complex-valued multiplication is deemed to be equivalent to four real-valued multiplications.

The corresponding complexity per bit may be expressed, respectively, as Eqs. (33)-(36), which are shown at the top of the following page. Here, the coherence block interval 


$$
\begin{aligned}
C_{\text {Mesleh }} & =\frac{(4 M N T Q+2 N T Q) / \tau+4 N T Q+2 Q+4 \mathcal{L}}{\log _{2}(Q \cdot \mathcal{L})}, \\
C_{\mathrm{ML}} & =\frac{(4 M N T Q+4 N T Q \mathcal{L}) / \tau+2 N T Q \mathcal{L}}{\log _{2}(Q \cdot \mathcal{L})}, \\
C_{\mathrm{I}} & =\frac{\left(4 M N T Q+4 N T Q+Q \mathcal{L}^{\prime}\right) / \tau+4 N T Q+3 Q \mathcal{L}^{\prime}+4 \mathcal{L}}{\log _{2}(Q \cdot \mathcal{L})}, \\
C_{\mathrm{II}} & =\frac{(4 M N T Q+4 N T Q) / \tau+4 N T Q+2 V Q+4 \mathcal{L}}{\log _{2}(Q \cdot \mathcal{L})}
\end{aligned}
$$

$\tau$ is defined as the number of space-time blocks, where the channel matrix $\mathbf{H}$ remains constant. This indicates that for example $\tau=1$ represents an instantaneously fading scenario and that upon increasing the coherence interval $\tau$, the associated complexity of Eqs. (33)-(36) may decrease, because for example the calculations of $\overline{\mathbf{H}}, s_{l} \overline{\mathbf{h}}_{q},\left\|\overline{\mathbf{h}}_{q}\right\|^{2}$ and $\mathbf{H}^{\prime}$ can be reused. Not that the complexity evaluations of the proposed Detectors I and II, as formulated in Eqs. (35) and (36), are detailed in the Appendix.

\section{F. Effects of Constellations on High-Q STSK Arrangements}

In this section, we provide a qualitative analysis of the effects of the specific constellation on the achievable coding gain of the QAM-aided STSK scheme.

Let us define the Pairwise Error Probability (PEP) as $P\left(\mathbf{S}_{l, q} \rightarrow \mathbf{S}_{l^{\prime}, q^{\prime}}\right)$, which quantifies the probability that a codeword $\mathbf{S}_{l, q}$ is erroneously decoded as $\mathbf{S}_{l^{\prime}, q^{\prime}}$. According to [21], at high SNRs the Chernoff bound of the PEP may be expressed as

$$
P\left(\mathbf{S}_{l, q} \rightarrow \mathbf{S}_{l^{\prime}, q^{\prime}}\right) \leq \underbrace{\left(\frac{1}{4 N_{0}}\right)^{R N}}_{\text {diversity gain }} \cdot \underbrace{\left(\prod_{r=1}^{R} \frac{1}{\mu_{r}}\right)^{N}}_{\text {coding gain }},
$$

where we have

$$
\begin{aligned}
\Delta & =\mathbf{S}_{l, q}-\mathbf{S}_{l^{\prime}, q^{\prime}} \\
R & =\operatorname{rank}\left(\Delta \Delta^{H}\right) .
\end{aligned}
$$

Furthermore, $\mu_{r}$ represents the $r$ th non-zero eigenvalue of $\Delta \Delta^{H}$. Assuming that codewords are designed to satisfy a rank of $R=\min (M, T)$, the performance difference is essentially determined by the minimum value of the multiplicative coding-gain term in Eq. (37) for the legitimate combinations of $\left(\mathbf{S}_{l, q}, \mathbf{S}_{l^{\prime}, q^{\prime}}\right)$. For simplicity, let us consider a scenario of $M=T$. Then, the coding gain $\left(\prod_{r=1}^{R} \frac{1}{\mu_{r}}\right)^{N}$ of Eq. (37) corresponds to $\operatorname{det}\left(\Delta \Delta^{H}\right)^{N}$, which may be classified by the relationship between $(l, q)$ and $\left(l^{\prime}, q^{\prime}\right)$ as Eqs. (40a)-(40c), which are shown at the top of the next page. Typically, the minimum value of $\operatorname{det}\left(\Delta \Delta^{H}\right)$ may be given by Eq. (40a) or Eq. (40b), rather than by Eq. (40c). We note, furthermore, that $\left|s_{l}-s_{l^{\prime}}\right|$ in Eq. (40a) corresponds to the distance between the constellation points $l$ and $l^{\prime}$, while $\left|s_{l}\right|$ indicates the absolute
TABLE II

COMPARISON OF SQUARE-QAM AND STAR-QAM CONSTELLATIONS

\begin{tabular}{|l|cc|}
\hline & $\begin{array}{c}\text { Minimum of } \\
\left|s_{l}\right|^{2}\end{array}$ & $\begin{array}{c}\text { Minimum of } \\
\left|s_{l}-s_{l^{\prime}}\right|^{2}\end{array}$ \\
\hline \hline 16 square-QAM & 0.2 & 0.4 \\
16 star-QAM [19] & 0.416 & 0.244 \\
\hline 32 square-QAM & 0.077 & 0.154 \\
32 star-QAM & 0.133 & 0.078 \\
\hline 64 square-QAM & 0.048 & 0.095 \\
64 star-QAM [20] & 0.279 & 0.042 \\
\hline
\end{tabular}

value of the $l$ th constellation point. ${ }^{7}$

For high $Q$ values it becomes more challenging to achieve a high value for $\operatorname{det}\left[\left(\mathbf{A}_{q}-\mathbf{A}_{q^{\prime}}\right)\left(\mathbf{A}_{q}-\mathbf{A}_{q^{\prime}}\right)^{H}\right]$ for all the legitimate $\left(q, q^{\prime}\right)$ combinations in Eq. (40b), than to maintain a high minimum value for $\operatorname{det}\left(\mathbf{A}_{q} \mathbf{A}_{q}^{H}\right)$ for all $q$ in Eq. (40a). Hence employing specific modulation schemes, which have a high minimum value of $\left|s_{l}\right|$, may result in a high coding gain. For example, let us compare in Table II two important constellations, namely 16 square-QAM [18] and 16 star-QAM [19]. Observe in Table II that star-QAM attains a higher value of $\left|s_{l}\right|$ than square-QAM for $\mathcal{L}=16,32$ and 64 . Hence somewhat unexpectedly, the star-QAM assisted STSK scheme has the potential of outperforming its square-QAM counterpart for high- $Q$ scenarios. This will be verified later in Section V.

\section{REduCED-COMPLEXITY NON-COHERENT STSK RECEIVER}

In this section, we firstly formulate the QAM-aided DSTSK scheme by further developing the DSTSK architecture of [11], [14]. Then, we apply the proposed reduced-complexity detectors of Sections III-C and III-D to our QAM-aided DSTSK receiver.

\section{A. System Model of QAM-Aided DSTSK Scheme}

We arrange for $Q$ dispersion matrices $\mathbf{B}_{q} \in \mathcal{C}^{T \times T}(q=$ $1, \cdots, Q)$ to be pre-assigned at the transmitter, similarly to the CSTSK scheme, where $\mathbf{B}_{q}$ is a unitary-matrix, which satisfies the relationship of $\mathbf{B}_{q} \mathbf{B}_{q}^{H}=\mathbf{B}_{q}^{H} \mathbf{B}_{q}=\mathbf{I}$. It is also assumed for the sake of simplicity that the number of transmit antennas

\footnotetext{
${ }^{7}$ To elaborate a little further, in the classic QAM modems the minimum distance $\min \left(\left|s_{l}-s_{l^{\prime}}\right|\right)$ affects the achievable performance, while the minimum absolute value $\min \left(\left|s_{l}\right|\right)$ does not. On the other hand, the recent STSK scheme does not obey this well-known rule, as mentioned above. For this reason, the star-QAM aided STSK scheme tends to outperforms its squareQAM counterpart, which is in contrast to classic QAM modems.
} 


$$
\operatorname{det}\left(\Delta \Delta^{H}\right)= \begin{cases}\left|s_{l}-s_{l^{\prime}}\right|^{2} \operatorname{det}\left(\mathbf{A}_{q} \mathbf{A}_{q}^{H}\right), & \text { for } l \neq l^{\prime}, q=q^{\prime} \\ \left|s_{l}\right|^{2} \operatorname{det}\left[\left(\mathbf{A}_{q}-\mathbf{A}_{q^{\prime}}\right)\left(\mathbf{A}_{q}-\mathbf{A}_{q^{\prime}}\right)^{H}\right], & \text { for } l=l^{\prime}, q \neq q^{\prime} \\ \operatorname{det}\left[\left(s_{l} \mathbf{A}_{q}-s_{l^{\prime}} \mathbf{A}_{q^{\prime}}\right)\left(s_{l} \mathbf{A}_{q}-s_{l^{\prime}} \mathbf{A}_{q^{\prime}}\right)^{H}\right], & \text { for } l \neq l^{\prime}, q \neq q^{\prime}\end{cases}
$$

$M$ is the same as the number of symbol durations $T$ per transmission block.

In each block duration, $B$ information bits are input and S/P converted to $B_{1}$ and $B_{2}$ bits, similarly to the CSTSK transmitter. According to the $B_{1}$ bits, one out of $Q$ dispersion matrices is activated as $\mathbf{B}_{q}^{(n)}$, while the $B_{2}$ bits are mapped to a PSK/QAM symbol $s_{l}^{(n)}$. Here, the superscript $n$ denotes the space-time block index. Finally, the transmitted space-time matrix $\mathbf{S}^{(n)}$ of the $n$th block may be formulated with the aid of differential encoding as follows:

$$
\mathbf{S}^{(n)}=\frac{s_{l}^{(n)}}{\left|s_{l}^{(n-1)}\right|} \mathbf{S}^{(n-1)} \mathbf{B}_{q}^{(n)} \quad(n \geq 1),
$$

where we have $\mathbf{S}^{(0)}=\mathbf{I}$ and $\left|s_{l}^{(0)}\right|=1 .^{8}$ Furthermore, the number of successively encoded blocks per frame is defined as $\zeta$ in this paper.

Assuming that the channel matrix $\mathbf{H}$ remains constant over two successive block intervals, the corresponding received signals at the $(n-1)$ st and the $n$th blocks may be expressed as

$$
\begin{aligned}
\mathbf{Y}^{(n-1)} & =\mathbf{H} \mathbf{S}^{(n-1)}+\mathbf{N}^{(n-1)} \\
\mathbf{Y}^{(n)} & =\mathbf{H} \mathbf{S}^{(n)}+\mathbf{N}^{(n)} \\
& =\frac{s_{l}^{(n)}}{\left|s_{l}^{(n-1)}\right|} \mathbf{H} \mathbf{S}^{(n-1)} \mathbf{B}_{q}^{(n)}+\mathbf{N}^{(n)} .
\end{aligned}
$$

From Eqs. (41), (42) and (44), we arrive at

$$
\mathbf{Y}^{(n)}=\frac{s_{l}^{(n)}}{\left|s_{l}^{(n-1)}\right|} \mathbf{Y}^{(n-1)} \mathbf{B}_{q}^{(n)}+\mathbf{N}^{\prime(n)},
$$

where we have the equivalent noise components of

$$
\mathbf{N}^{\prime(n)}=\mathbf{N}^{(n)}-\frac{s_{l}^{(n)}}{\left|s_{l}^{(n-1)}\right|} \mathbf{N}^{(n-1)} \mathbf{B}_{q}^{(n)} .
$$

Hence, the ML detection at the $n$th block interval may be represented by

$$
(\hat{q}, \hat{l})=\arg \min _{(q, l)}\left\|\mathbf{Y}^{(n)}-\frac{s_{l}^{(n)}}{\nu} \mathbf{Y}^{(n-1)} \mathbf{B}_{q}^{(n)}\right\|,
$$

where we assume that the estimate $\nu=\left|s_{\hat{l}}^{(n-1)}\right|$ is obtained from the ML detection result of the $(n-1)$ st block, acknowledging that this may potentially induce error propagation to the detection of the $n$th block. We also note that the DSTSK scheme's ML detector formulated in Eq. (47) does not

\footnotetext{
${ }^{8}$ Although we assumed for the QAM-aided DSTSK scheme to have the relationship of $M=T$, a lower transmit-AE scenario of $M<T$ may be readily used by choosing $M<T$ rows from the signals $\mathbf{S}^{(n)}$ in Eq. (41). This implementation is similar to that of the $G_{3}$-OSTBC [22].
}

include any channel elements, hence dispensing with channel estimation.

\section{B. The Proposed DSTSK Detector}

In order to enable us to implement the reduced-complexity MF detectors proposed for the CSTSK scheme in the context of the above-mentioned QAM-aided DSTSK scheme, we firstly derive a linearized DSTSK model, which corresponds to that of the CSTSK scheme of Eq. (2). More specifically, by applying the vectorial stacking operation $\operatorname{vec}(\cdot)$ at both sides of Eq. (45), we get

$$
\overline{\mathbf{Y}}^{(n)}=\overline{\mathbf{H}}^{(n)} \mathbf{K}_{q, l}^{(n)}+\overline{\mathbf{N}}^{(n)},
$$

where we have

$$
\begin{aligned}
\overline{\mathbf{Y}}^{(n)} & =\operatorname{vec}\left(\mathbf{Y}^{(n)}\right), \\
\overline{\mathbf{H}}^{(n)} & =\left(\mathbf{I}_{T} \otimes \frac{\overline{\mathbf{Y}}^{(n-1)}}{\left|s_{l}^{(n-1)}\right|}\right) \chi, \\
\chi & =\left[\operatorname{vec}\left(\mathbf{B}_{1}\right), \cdots, \operatorname{vec}\left(\mathbf{B}_{Q}\right)\right], \\
\overline{\mathbf{N}}^{(n)} & =\operatorname{vec}\left(\mathbf{N}^{\prime(n)}\right)
\end{aligned}
$$

and

$$
\begin{gathered}
\mathbf{K}_{q, l}^{(n)}=\left[0, \cdots, 0, s_{l}^{(n)}, 0, \cdots, 0\right]^{\mathrm{T}} \\
\uparrow \\
q \text { th element }
\end{gathered}
$$

Since Eq. (48) obeys the same system model as Eq. (2), the detectors proposed in Sections III-C and III-D can be directly applied to our PSK/QAM-aided DSTSK scheme. This implies that higher-order PSK/QAM constellations can be readily employed in a similar manner to the CSTSK scenario, while those of the previous DSTSK scheme [14] are limited to constellations up to 8-PSK.

Again, as shown in Eq. (50), the matrix $\overline{\mathbf{H}}^{(n)}$ does not contain any channel elements. Additionally, unlike the CSTSK scenarios of Section III-C, the proposed DSTSK detector has to update $\overline{\mathbf{H}}^{(n)}$ at each block interval, regardless of the channel's coherence time $\tau$. By contrast, while the performance of the CSTSK scheme is routinely degraded by channel estimation errors, the proposed DSTSK detector allows us to dispense with channel estimation, hence potentially outperforming its CSTSK counterpart.

We also note that our DSTSK detector has the explicit benefit of dispensing with the channel power estimation, while this would be necessary for the QAM-aided Differential Orthogonal Space-Time Block Codes (OSTBC) detector of [23]. This contributes towards the mitigation of error propagation to the forthcoming signaling blocks. 

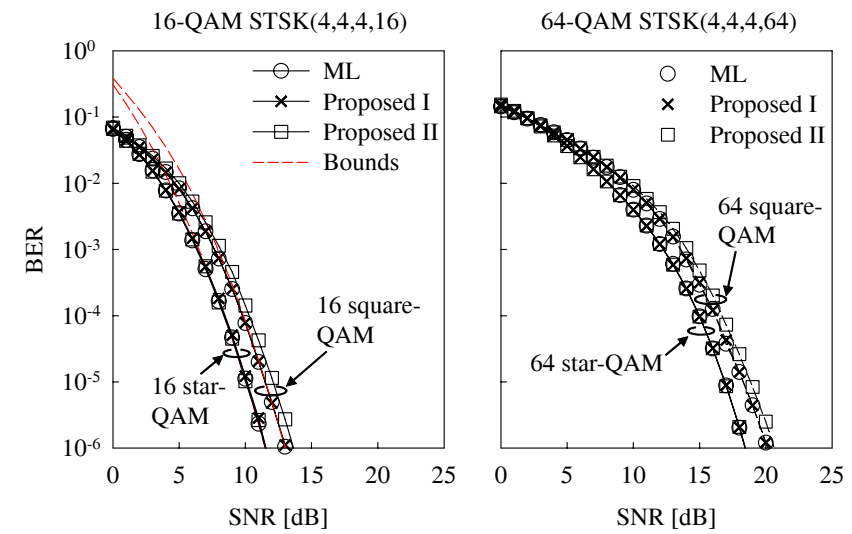

Fig. 3. Achievable BER performance of the 16-QAM CSTSK $(4,4,4,16)$ and 64-QAM CSTSK $(4,4,4,64)$ schemes, where the optimal ML detector as well as the proposed Detectors I and II were considered, while comparing starand square-QAM modulations.

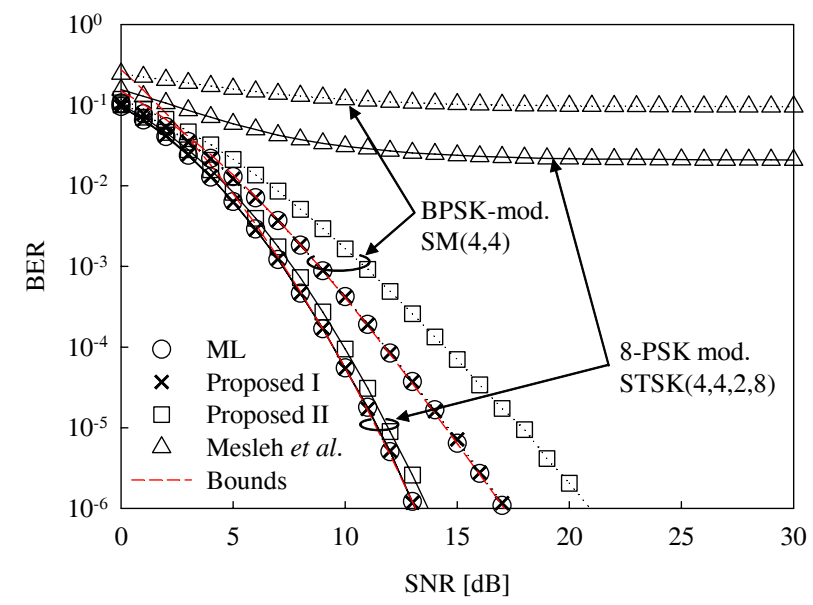

Fig. 4. Achievable BER performance of the 8-PSK modulated $\operatorname{CSTSK}(4,4,2,8)$ scheme and the BPSK-modulated SM scheme employing $M=N=4$ transmit and receive antennas, where the optimal ML detector, the proposed Detectors I and II and Mesleh's MF-based detector were considered. Here, all the arrangements have the throughput of 3 bits/symbol and are equipped with $M=N=4$ transmit and receive antennas.

\section{Performance Results}

In this section we provide our performance results for characterizing both the achievable BER and the computational complexity of the above-mentioned four detectors. ${ }^{9}$ Throughout the simulations, we considered frequency-flat block Rayleigh fading channels.

In order to generate a dispersion-matrix set for each STSK arrangement, Monte Carlo simulations were carried out, where the dispersion-matrix set was optimized according to the rankand determinant criterion [11], by generating $10^{5}-10^{6}$ random matrices. Additionally, for the CSTSK and DSTSK schemes, having the relationship of $M=T$, we imposed a unitarymatrix constraint on the randomly generated matrices.

\footnotetext{
${ }^{9}$ Since the performance advantage of the class of STSK schemes over other MIMO arrangements has been shown in the previous studies of [11], [13], [24], in this paper we focus our attaintion on the comparisons between the proposed and conventional STSK detectors. For readers who are interested in the performance differences between the STSK scheme and the conventional MIMO arrangements, please refer to [11], [13], [24]
}

Figs. 3 and 4 compare the BER of the STSK family, namely of the SM and the CSTSK schemes, employing $M=4$ transmit and $N=4$ receive antenna elements. In Fig. 3 we considered the 16-QAM assisted $\operatorname{CSTSK}(4,4,4,16)$ and 64-QAM assisted CSTSK $(4,4,4,64)$ schemes, employing square- and star-QAM constellations, while in Fig. 4 the BER curves of the BPSK-modulated SM and the 8-PSK modulated $\operatorname{CSTSK}(4,4,2,8)$ schemes having $R=3$ bits/symbol were portrayed. Here, we also plotted the corresponding tight BER upper-bound curves calculated based on the MomentGenerating Function (MGF) [25], in order to confirm the ML detector's BER results.

Observe in Fig. 3 that both the $\mathcal{L}=16$ and 64 scenarios showed the similar results. More specifically, for star-QAM the three detectors, i.e. the ML detector and the proposed Detectors I and II, exhibited the alomost identical performance, while for square-QAM Detector II showed a fraction of one $\mathrm{dB}$ worse performance than those of the ML detector and Detector I. This marginal performance penalty of Detector II is owing to the fact that Detector II is designed to approximate the $q$-estimation part of Detector I for the sake of substantially reducing the complexity, whose effects will also be verified later in our simulations.

Also, in Fig. 4 while the original MF detector exhibited an error floor as predicted from [2], [10], the proposed Detector II achieved a near-optimal performance for CSTSK, similarly to the results in Fig. 3.

In order to provide further insights, in Fig. 5 we compared the effective SNRs recorded for BER $=10^{-4}$ in the context of the 16-square-QAM and 16-star-QAM aided $\operatorname{CSTSK}(4,4, T, Q)$ schemes employing both the ML and the proposed MF Detectors I and II. More specifically, in Fig. 5(a) we varied the number of dispersion matrices from $Q=1$ to 64 , while maintaining the number of symbol durations at $T=4$. Furthermore, in Fig. 5(b) we varied the number of symbol durations per block as $T=1,2,3,4$ and 5 . Observe in Fig. 5(a) that since an increase of $Q$ increases the normalized throughput, this naturally increased the effective SNR required for both QAM modems. The findings from Figs. 5(a) and 5(b) are listed below:

- As predicted from Section III-F, the 16-star-QAM aided CSTSK scheme outperformed its 16-square-QAM aided counterpart for $Q \geq 2$ and its performance advantage increased upon increasing the value of $Q$ regardless of the specific choice of the detectors employed.

- The ML detector and Detector I exhibited an identical performance for all the scenarios considered.

- For $M \leq T$ 16-star-QAM scenarios the proposed reduced-complexity MF Detector II achieved a performance comparable to that of the optimal ML detector, whilst there was an observable performance gap between Detector II and the other two detectors for 16-squareQAM and for 16-star-QAM scenarios associated with $M>T .{ }^{10}$

- Observe in Fig. 5(b) that the lower the value of $T$, the

\footnotetext{
${ }^{10}$ An information-theoretic proof of the proposed detector's capability of attaining the exact scenario-specific ML performance remains an open issue, which will be investigated in our future studies.
} 


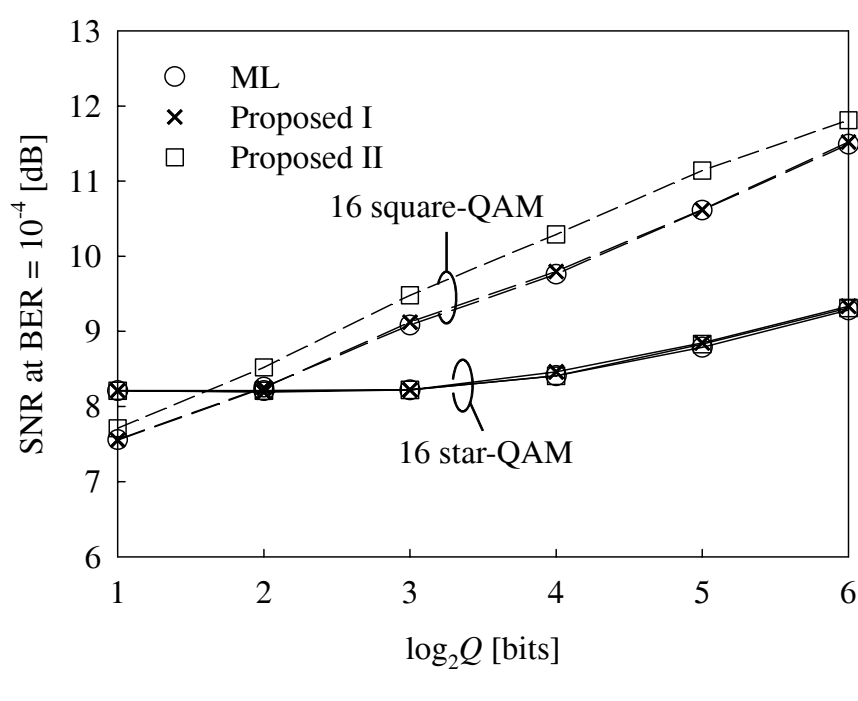

(a) $T=4$

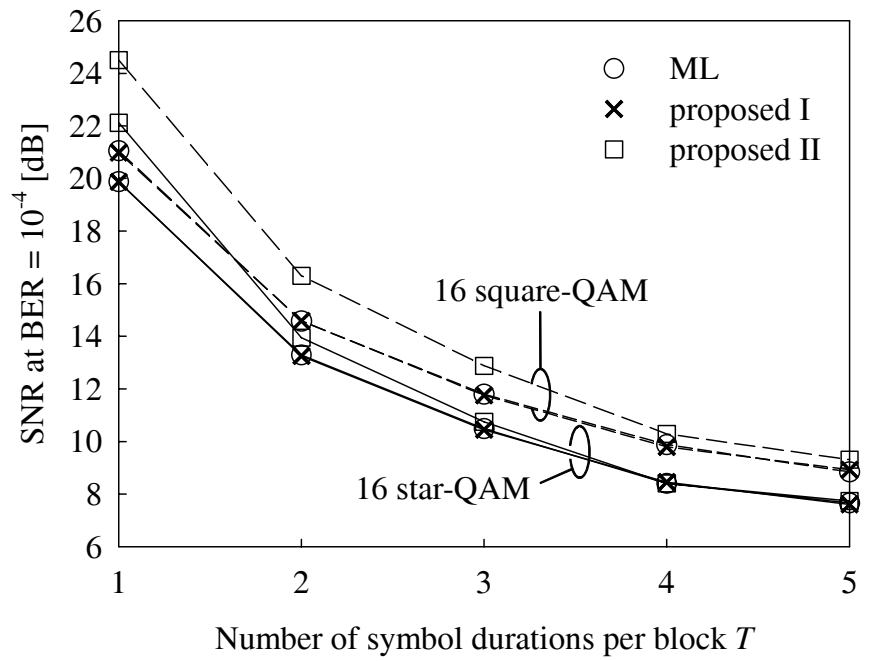

(b) $Q=16$

Fig. 5. Comparison of effective SNRs recorded for BER $=10^{-4}$ between the 16-square-QAM and 16-star-QAM aided CSTSK $(4,4, T, Q)$ schemes employing the ML detector as well as the proposed Detector I and Detector II. (a) $\log _{2} Q=1-6, T=4$ and (b) $T=1-5, Q=16$.
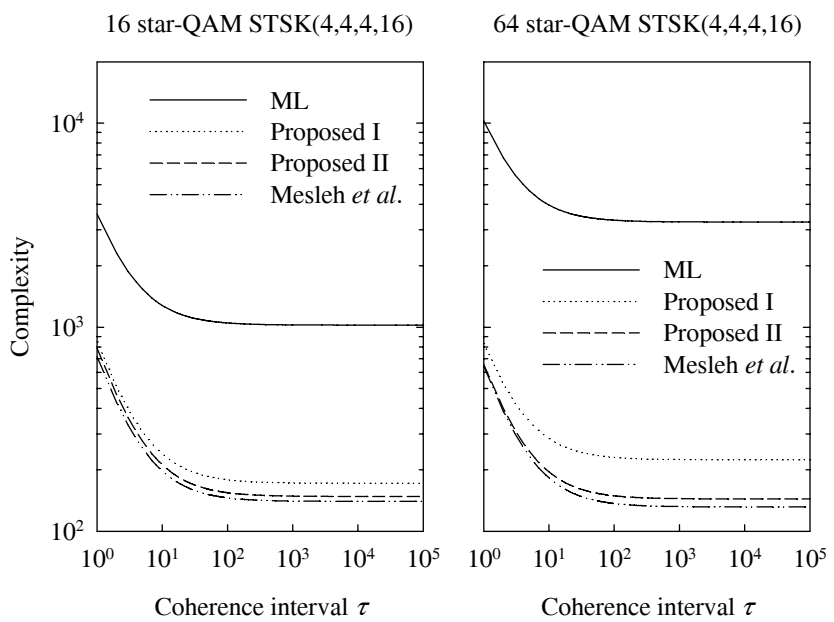

Fig. 6. Complexity comparison between the four detectors, which are presented in Section III, for the 16-star-QAM as well as 64-star-QAM assisted $\operatorname{CSTSK}(4,4,4,16)$ arrangements.

higher the performance gap became between Detector II and the other two detectors.

Hence, it may be concluded that star-QAM constellations may be more suitable for the high- $Q$ CSTSK scheme than the classic square-QAM constellations. Let us emphasize that this conclusion is somewhat surprising, since it is different from the conventional QAM modems.

Moreover, Fig. 6 shows the computational complexity imposed by the above-mentioned four detectors for the 16-star as well as 64-star-QAM assisted $\operatorname{CSTSK}(4,4,4,16)$ schemes. Observe in Fig. 6 that upon increasing the coherence interval $\tau$, the complexity of each detector was reduced towards a certain minimum value. As expected, the conventional MF detector as well as the Detectors I and II attained a significantly lower complexity than that of the ML detector. To be more specific, the conventional MF detector and Detector II exhibited almost the same complexity, which is approximately

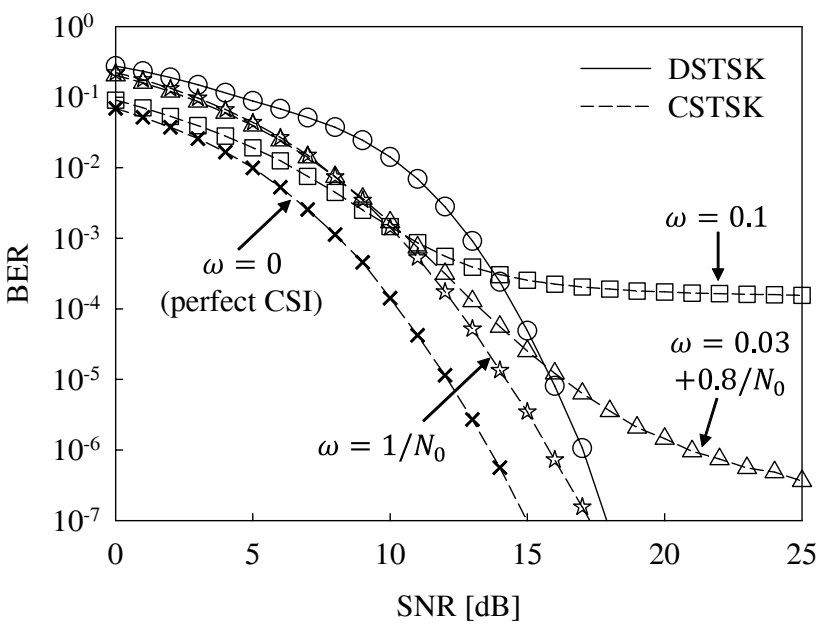

Fig. 7. BER comparison of the 16-square-QAM aided $\operatorname{CSTSK}(4,4,4,16)$ and the 16-star-QAM aided DSTSK $(4,4,4,16)$ schemes, where the proposed MF-based Detector II was employed for both the schemes. We also considered the effects of CSI error associated with an equivalent channel estimation's noise variance of $\omega=0,0.1,0.03+0.8 / N_{0}$ and $1 / N_{0}$. The number of successive blocks $\zeta$ for the DSTSK scheme was set to $\zeta=10$.

twice lower than that of Detector I for the 64-QAM scenario.

In Fig. 7 we compared the achievable BER performance of the 16-square-QAM aided CSTSK $(4,4,4,16)$ and the 16-starQAM aided DSTSK $(4,4,4,16)$ schemes, both achieving the normalized throughput of $2 \mathrm{bits} / \mathrm{symbol}$, where the proposed MF-based Detector II was employed for both the schemes. Here, we also considered the effects of CSI errors, where the estimated channels were contaminated by the additive Gaussian noise of $\mathcal{C N}(0, \omega)$ having a power of $\omega=0,0.1$ and $0.03+0.8 / N_{0} \mathrm{~dB}[26]$ as well as $\omega=1 / N_{0}$ in comparison to the average signal power. Observe in Fig. 7 that although the CSTSK scheme outperformed its DSTSK counterpart for the perfect-CSI and $\omega=1 / N_{0}$ scenarios, upon introducing CSIestimation errors, it exhibited an error floor. This is because 
the DSTSK scheme remained unaffected by the potential CSI-estimation errors owing to the explicit benefit of noncoherent detection. Other implicit benefits of the DSTSK scheme are that it does not require any pilot overhead and that is completely dispensing with the complexity associated with channel estimation unlike its CSTSK counterpart.

Next, we compared in Fig. 8 the achievable BER performance of the 16-star-QAM and 16-square-QAM $\operatorname{DSTSK}(4,4,4,16)$ schemes, where the number of successive blocks $\zeta$ per frame was varied as $\zeta=5,10,25,50$ and 100. It can be seen from Fig. 8 that an increase of $\zeta$ leads to a performance degradation for both schemes, since the mis-detection of $s_{l}^{(n-1)}$ at the $(n-1)$ st block affects the following block, as shown in Eq. (47). However, the degradation was found to be marginal, especially for high SNRs. Surprisingly, the 16 starQAM scenario tended to attain a better performance than that of 16 square-QAM, similarly to the CSTSK systems. This is owing to the fact that the system model of the DSTSK scheme has the same structure as that of the CSTSK scheme, as shown in Eqs. (2) and (48). Hence the discussions of Section III-F are also valid for the DSTSK scheme.

Based on the results of our simulations, it was found that the proposed Detectors I and II have their own performance versus complexity tradeoffs, depending on the particular STSK scenarios considered. More specifically, the following guidelines may be provided:

- For the $M \leq T$ STSK schemes employing star-QAM or PSK modulations, the adoption of Detector II is preferable to that of Detector I, since the complexity of Detector II is lower than that of Detector I, while both the proposed schemes are capable of attaining the optimal ML performance.

- For other scenarios, we have an option of choosing either of the proposed Detectors I or II, according to the receiver's design policy regarding its decoding complexity and the achievable performance. This is because the BER performance of Detector I is marginally better than that of Detector II, while Detector II's decoding complexity is lower than that of Detector I.

Again, although predominantly the exhaustive ML search has been employed for the SM, SSK and STSK schemes, the proposed reduced-complexity detectors have the potential of replacing it without any substantial performance loss.

\section{CONCLUSIONS}

In this paper, we proposed a reduced-complexity nearoptimal detector for the STSK scheme employing an arbitrary PSK/QAM constellation, which exploits the STSK-specific IEI-free system model, rather than that of spatial multiplexing. More specifically, the proposed MF detector takes into account the specific constellation diagram considered. As a result, our detector is capable of achieving a lower complexity than that of ML detection, while avoiding any substantial BER performance loss. Interestingly, our simulation results demonstrated that the proposed reduced-complexity detector may achieve a performance identical to that of the optimal ML detector for the specific STSK's parameters. Therefore, the employment of this detector further augments the benefits

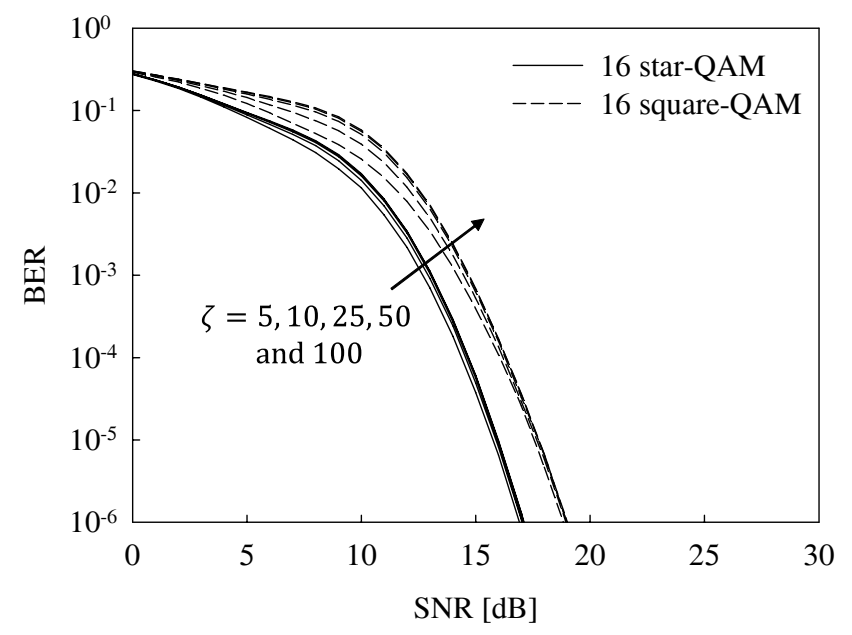

Fig. 8. Achievable BER performance of the 16-star-QAM and 16-squareQAM DSTSK $(4,4,4,16)$ schemes employing the proposed Detector II, where the number of successive blocks $\zeta$ per frame was varied from $\zeta=5$ to 100 .

of the CSTSK scheme. Furthermore, we generalized the previous PAM- or PSK-aided DSTSK concept to conceive its more bandwidth-efficient QAM-aided counterpart. Then, the proposed reduced-complexity CSTSK detector was applied to the QAM-aided DSTSK scheme, which enabled us to carry out low-complexity non-coherent detection, while dispensing with any channel estimation as well as eliminating the pilot overhead. Moreover, it was found from our theoretical and numerical analysis that the star-QAM assisted STSK scheme tends to outperform its square-QAM counterpart, especially for high- $Q$ scenarios.

The proposed detector designed for the class of co-located STSK schemes readily lends itself to cooperative communications [27], [28] as well as relying on semi-blind joint channel estimation and data detection [12].

\section{APPENDIX}

The complexity of the proposed MF-based Detectors I and II is represented by Eqs. (35) and (36), respectively. This appendix provides more detailed information for this assessment.

\section{A. The Complexity of Detector I}

Firstly, we assume that the receiver stores the power of each constellation point $\left|s_{l}\right|^{2}(l=1, \cdots, \mathcal{L})$. After estimating the channels $\mathbf{H}$, the equivalent channels $\overline{\mathbf{H}}=\left(\mathbf{I}_{T} \otimes \mathbf{H}\right) \chi$ of Eq. (4) are calculated. The associated complexity is given by

$$
\operatorname{comp}\left[\left(\mathbf{I}_{T} \otimes \mathbf{H}\right) \chi\right]=4 M N T Q,
$$

where 'comp[.]' represents the number of real-valued multiplications, which is required for calculating ' $'$ '. Then, the norm of each column of $\overline{\mathbf{H}}$, namely $\left\|\overline{\mathbf{h}}_{q}\right\|(q=1, \cdots, Q)$, is computed at a complexity of

$$
\operatorname{comp}\left[\left\|\overline{\mathbf{h}}_{q}\right\|(q=1, \cdots, Q)\right]=2 N T Q .
$$

Then the calculation of $\mathbf{H}^{\prime}=\left[\overline{\mathbf{h}}_{1} /\left\|\overline{\mathbf{h}}_{1}\right\| \cdots \overline{\mathbf{h}}_{Q} /\left\|\overline{\mathbf{h}}_{Q}\right\|\right]$ in Eq. (13) imposes a complexity of

$$
\operatorname{comp}\left[\mathbf{H}^{\prime}\right]=2 N T Q \text {. }
$$


Since Eqs. (54), (55) and (56) only have to be updated at intervals determined by the channel's coherence time $\tau$, the average complexity may be expressed as

$$
\frac{4 M N T Q+4 N T Q}{\tau} \text {. }
$$

Furthermore, the MF operation of Eq. (14) imposes a complexity of

$$
\operatorname{comp}\left[\mathbf{H}^{\prime H} \overline{\mathbf{Y}}\right]=4 N T Q,
$$

while the (l.q) detection of Eq. (21) has a complexity of Eqs. (59) and (60). Finally, considering that each block of the STSK scheme carries $\log _{2}(Q \cdot \mathcal{L})$ bits, the total per-bit complexity of the proposed Detector I is given by Eq. (35), also taking into account Eqs. (57), (58), (59) and (60). See Eqs. (59) and (60) at the top of the next page.

\section{B. The Complexity of Detector II}

By contrast, in the proposed Detector II the detection of dispersion-matrix $q$, as formulated in Eq. (21), is replaced by the approximated version of Eq. (26), whose complexity is given by

$$
\operatorname{comp}\left[\arg \max _{q, \forall v}\left[\left|\Re\left(z_{q}\right)\right| x_{v, I}+\left|\Im\left(z_{q}\right)\right| x_{v, Q}\right]\right]=2 V Q .
$$

Hence, based on Eqs. (57), (58), (60) and (61), we arrive at the complexity of Detector II as in Eq. (36).

\section{REFERENCES}

[1] R. Y. Mesleh, H. Haas, S. Sinanovic, C. Ahn, and S. Yun, "Spatial modulation," IEEE Trans. Veh. Technol., vol. 57, no. 4, pp. 2228-2242, 2008.

[2] J. Jeganathan, A. Ghrayeb, and L. Szczecinski, "Spatial modulation: optimal detection and performance analysis," IEEE Commun. Lett., vol. 12 , no. 8, pp. 545-547, 2008.

[3] N. Serafimovski, M. Di Renzo, S. Sinanovic, R. Y. Mesleh, and H. Haas, "Fractional bit encoded spatial modulation (FBE-SM)," IEEE Commun. Lett., vol. 14, no. 5, pp. 429-431, 2010.

[4] R. Y. Mesleh, M. Di Renzo, H. Haas, and P. M. Grant, "Trellis coded spatial modulation," IEEE Trans. Wireless Commun., vol. 9, no. 7, pp. 2349-2361, 2010.

[5] J. Jeganathan, A. Ghrayeb, L. Szczecinski, and A. Ceron, "Space shift keying modulation for MIMO channels," IEEE Trans. Wireless Commun., vol. 8, no. 7, pp. 3692-3703, 2009.

[6] M. Di Renzo and H. Haas, "Improving the performance of space shift keying (SSK) modulation via opportunistic power allocation," IEEE Commun. Lett., vol. 14, no. 6, pp. 500-502, 2010.

[7] — "A general framework for performance analysis of space shift keying (SSK) modulation for MISO correlated Nakagami- $m$ fading channels," IEEE Trans. Commun., vol. 58, no. 9, pp. 2590-2603, 2010.

[8] G. Foschini and M. Gans, "On limits of wireless communications in a fading environment when using multiple antennas," Wireless Pers. Commun., vol. 6, no. 3, pp. 311-335, 1998.

[9] P. Wolniansky, G. Foschini, G. Golden, and R. Valenzuela, "V-BLAST: an architecture for realizing very high data rates over the rich-scattering wireless channel," in Proc. Int. Symp. Signals, Systems, Electronics, 1998, pp. 295-300.

[10] J. Jeganathan, A. Ghrayeb, and L. Szczecinski, "Generalized space shift keying modulation for MIMO channels," in Proc. IEEE 19th Int. Symp. Personal, Indoor Mobile Radio Commun., Sep. 2008, pp. 1-5.

[11] S. Sugiura, S. Chen, and L. Hanzo, "Coherent and differential spacetime shift keying: a dispersion matrix approach," IEEE Trans. Commun., vol. 58, no. 11, pp. 3219-3230, Nov. 2010

[12] S. Chen, S. Sugiura, and L. Hanzo, "Semi-blind joint channel estimation and data detection for space-time shift keying systems," IEEE Signal Process. Lett., vol. 17, no. 12, pp. 993-996, Dec. 2010.
[13] S. Sugiura, S. Chen, and L. Hanzo, "Generalized space-time shift keying designed for flexible diversity-, multiplexing- and complexity-tradeoffs," IEEE Trans. Wireless Commun., vol. 10, no. 4, pp. 1144-1153, Apr. 2011.

[14] C. Xu, S. Sugiura, S. X. Ng, and L. Hanzo, "Reduced-complexity noncoherently detected differential space-time shift keying," IEEE Signal Process. Lett., vol. 18, no. 3, pp. 153-156, Mar. 2011.

[15] A. Younis, R. Mesleh, H. Haas, and P. Grant, "Reduced complexity sphere decoder for spatial modulation detection receivers," in Proc. IEEE Global Commun. Conf., Dec. 2010, pp. 1-5.

[16] L. Hanzo, O. Alamri, M. El-Hajjar, and N. Wu, Near-Capacity MultiFunctional MIMO Systems: Sphere-Packing, Iterative Detection and Cooperation. John Wiley and IEEE Press, 2009.

[17] L. Hanzo, Y. Akhtman, L. Wang, and M. Jiang, MIMO-OFDM for LTE, WIFI and WIMAX: Coherent Versus Non-Coherent and Cooperative Turbo-Tranceivers. John Wiley and IEEE Press, 2011.

[18] L. Hanzo, S. Ng, W. T. Webb, and T. Keller, Quadrature Amplitude Modulation: From Basics to Adaptive Trellis-Coded, Turbo-Equalised and Space-Time Coded OFDM, CDMA and MC-CDMA Systems. John Wiley and IEEE Press, 2004.

[19] X. Dong, N. Beaulieu, and P. Wittke, "Error probabilities of twodimensional $M$-ary signaling in fading," IEEE Trans. Commun., vol. 47, no. 3, pp. 352-355, 1999.

[20] T. May, H. Rohling, and V. Engels, "Performance analysis of Viterbi decoding for 64-DAPSK and 64-QAM modulated OFDM signals," IEEE Trans. Commun., vol. 46, no. 2, pp. 182-190, 1998.

[21] R. Heath Jr and A. Paulraj, "Linear dispersion codes for MIMO systems based on frame theory," IEEE Trans. Signal Process., vol. 50, no. 10, pp. 2429-2441, 2002.

[22] V. Tarokh, N. Seshadri, and A. Calderbank, "Space-time codes for high data rate wireless communication: performance criterion and code construction," IEEE Trans. Inf. Theory, vol. 44, no. 2, pp. 744-765, 1998.

[23] C. S. Hwang, S. H. Nam, J. Chung, and V. Tarokh, "Differential space time block codes using nonconstant modulus constellations," IEEE Trans. Signal Process., vol. 51, no. 11, pp. 2955-2964, 2005.

[24] S. Sugiura, S. Chen, and L. Hanzo, "A universal space-time architecture for multiple-antenna aided systems," IEEE Commun. Surveys Tutorials, 2012, doi: 10.1109/SURV.2011.041911.00105, in press.

[25] M. K. Simon and M. S. Alouini, Digital Communication over Fading Channels. Wiley-IEEE Press, 2005.

[26] T. Yoo and A. Goldsmith, "Capacity and power allocation for fading MIMO channels with channel estimation error," IEEE Trans. Inf. Theory, vol. 52, no. 5, pp. 2203-2214, 2006.

[27] S. Sugiura, S. Chen, and L. Hanzo, "Packet-reliability based decodeand-forward relaying aided distributed space-time shift keying," in Proc. IEEE Global Commun. Conf., Dec. 2010, pp. 1-5.

[28] S. Sugiura, S. Chen, H. Haas, P. M. Grant, and L. Hanzo, "Coherent versus non-coherent decode-and-forward relaying aided cooperative space-time shift keying," IEEE Trans. Commun., vol. 59, no. 6, pp. 1707-1719, June 2011

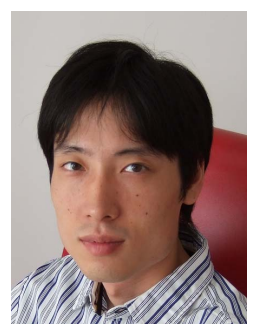

Shinya Sugiura (M'06) received the B.S. and M.S. degrees in aeronautics and astronautics from $\mathrm{Ky}$ oto University, Kyoto, Japan, in 2002 and 2004, respectively, and the $\mathrm{Ph} . \mathrm{D}$. degree in electronics and electrical engineering from the University of Southampton, Southampton, UK, in 2010. Since 2004, he has been with Toyota Central R\&D Laboratories, Inc., Japan. His research has covered a range of areas in communications, including space-time modulation/demodulation, turbo coding, cooperative communications, multiuser detection, and automotive antenna design, as well as vehicular ad hoc networking.

Dr. Sugiura has published over 40 research papers in various journals and conference proceedings. He was awarded the IEEE AP-S Japan Chapter Young Engineer Award in December 2008. 


$$
\begin{array}{r}
\operatorname{comp}\left[\arg \max _{q, \forall l^{\prime}}\left[2\left\|\overline{\mathbf{h}}_{q}\right\|\left\{\left|\Re\left(z_{q}\right)\right|\left|\Re\left(s_{l^{\prime}}^{\prime}\right)\right|+\left|\Im\left(z_{q}\right)\right|\left|\Im\left(s_{l^{\prime}}^{\prime}\right)\right|\right\}-\left\|\overline{\mathbf{h}}_{q}\right\|^{2}\left|s_{l^{\prime}}^{\prime}\right|^{2}\right]\right]=3 Q \mathcal{L}^{\prime}+\frac{Q \mathcal{L}^{\prime}}{\tau} \\
\operatorname{comp}\left[\arg \min _{l}\left|z_{\hat{q}}-\left\|\overline{\mathbf{h}}_{\hat{q}}\right\| s_{l}\right|\right]=4 \mathcal{L}
\end{array}
$$

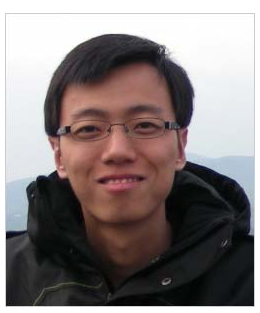

Chao Xu (S'09) received a B.Eng. degree from Beijing University of Posts and Telecommunications, Beijing, China, and a B.Sc. (Eng) with First Class Honours from Queen Mary, University of London, London, UK, through a Sino-UK joint degree program in 2008 , both in telecommunications engineering with management. In 2009, he obtained a M.Sc. degree with distinction in radio frequency communication systems from the University of Southampton, Southampton, UK, and he was awarded the IEEE Communications Society UK\&RI Chapter Best MSc Student in Broadband and Mobile Communication Networks. He is currently working towards the Ph.D. degree with the Communications Research Group, School of Electronics and Computer Science, University of Southampton, UK. His research interests include reduced-complexity MIMO design, noncoherent space-time modulation detection, and EXIT-chart-aided turbo detection, as well as cooperative communications.

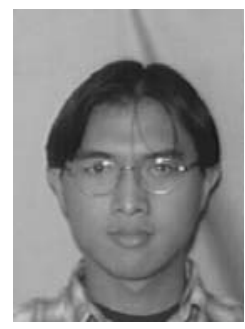

Soon Xin Ng (S'99-M'03-SM'08) received the B.Eng. degree (First Class) in electronics engineering and the Ph.D. degree in wireless communications from the University of Southampton, Southampton, U.K., in 1999 and 2002, respectively. From 2003 to 2006, he was a postdoctoral research fellow working on collaborative European research projects known as SCOUT, NEWCOM, and PHOENIX. Since August 2006, he has been a member of the academic staff in the School of Electronics and Computer Science, University of Southampton. He is involved in the OPTIMIX European project, as well as the IU-ATC and UC4G projects. His research interests include adaptive coded modulation, coded modulation, channel coding, space-time coding, joint source and channel coding, iterative detection, OFDM, MIMO, cooperative communications, and distributed coding. He has published over 120 papers and co-authored two John Wiley/IEEE Press books. He is a senior member of the IEEE and a fellow of the Higher Education Academy in the UK.

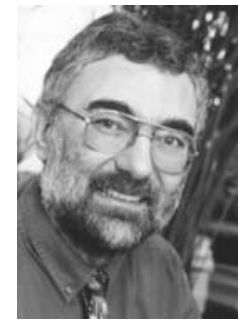

Lajos Hanzo (M'91-SM'92-F'04) FREng, FIEEE, FIET, Eurasip Fellow, DSc received his master's degree in electronics in 1976 and his doctorate in 1983. In 2010, he was awarded the 'Doctor Honaris Causa' honorary doctorate by the Technical University of Budapest. During his 35-year career in telecommunications he has held various research and academic posts in Hungary, Germany, and the UK. Since 1986, he has been with the School of Electronics and Computer Science, University of Southampton, UK, where he holds the chair in telecommunications. He has co-authored 20 John Wiley - IEEE Press books on mobile radio communications totalling in excess of 10,000 pages, published $1200+$ research papers and book chapters (which can be found on IEEE Xplore), acted as TPC Chair of IEEE conferences, presented keynote lectures, and has been awarded a number of distinctions. Currently, he is directing a 100 -strong academic research team, working on a range of research projects in the field of communications, signal processing, and control. The team is sponsored by industry, the Engineering and Physical Sciences Research Council (EPSRC) UK, the European IST Programme, and the Mobile Virtual Centre of Excellence (VCE), UK. He is an enthusiastic supporter of industry and an academic liaison, and he offers a range of industrial courses. $\mathrm{He}$ is also an IEEE Distinguished Lecturer as well as a Governor of the IEEE VTS. He is the Editor-in-Chief of the IEEE Press and a Chaired Prof. at Tsinghua University, Beijing. For further information on research in progress and associated publications please refer to http://www-mobile.ecs.soton.ac.uk. 